$\underline{\text { A short communication }}$

\title{
Understanding strong coupling constant, nuclear stability and binding energy with three atomic gravitational constants - A short communication
}

\author{
U.V.S. Seshavatharam ${ }^{1}$ and S. Lakshminarayana ${ }^{2}$ \\ ${ }^{1}$ Honorary faculty, I-SERVE, Survey no-42, Hitech city, Hyderabad-84,Telangana, INDIA \\ ${ }^{2}$ Dept. of Nuclear Physics, Andhra University, Visakhapatnam-03,AP, INDIA \\ Emails: seshavatharam.uvs@gmail.com (and) Insrirama@gmail.com \\ Orcid numbers : 0000-0002-1695-6037 (and) 0000-0002-8923-772X
}

\begin{abstract}
We present simple relations for nuclear stability and nuclear binding energy with respect to three gravitational constants associated with electroweak, strong and electromagnetic interactions.
\end{abstract}

\section{Introduction}

Considering neutrons and protons as microscopic molecules, the liquid drop model treats the atomic nucleus as a drop of incompressible nuclear fluid of very high density bound by strong nuclear force. The residual effect of the strong nuclear force plays a crucial role in understanding nuclear binding. Mathematical formula constitutes five different energy terms and five different energy coefficients. Energy coefficients are chosen in such a way to fit the wide range of nuclear binding energy data partly based on theory and partly based on empirical measurements. Hence 'liquid drop formula' is generally called as 'Semi empirical mass formula (SEMF). Even though, many scientists reviewed the formula in different ways, as on today, the syntax of the formula almost remains the same with very minor changes [1]. The inverse problem framework [1], allows to infer the underlying model parameters from experimental observation, rather than to predict the observations from the model parameters. Recently, the ground-state properties of nuclei with $Z=8$ to 120 from the proton drip line to the neutron drip line have been investigated using the spherical relativistic continuum HartreeBogoliubov (RCHB) theory [2] with the relativistic density functional PC-PK1.

In this context, we would like to emphasize the fact that, physics and mathematics associated with fixing of the energy coefficients of SEMF are neither connected with residual strong nuclear force nor connected with strong coupling constant. Since nuclear force is mediated via quarks and gluons, it is necessary and compulsory to study the nuclear binding energy scheme in terms of nuclear coupling constants. In this direction, N. Ghahramany and team members have taken a great initiative in exploring the secrets of nuclear binding energy and magic numbers [3]. Very interesting point of their study is that nuclear binding energy can be understood with two or three terms having single energy coefficient.

\section{Basic Ideas}

The four basic interactions can be allowed to have four different gravitational constants [4-12]. The three atomic gravitational constants help in understanding neutron-proton stability. Electromagnetic and nuclear gravitational constants play a role in understanding proton-electron mass ratio, Bohr radius and characteristic atomic radius. With reference to the weak gravitational constant, it is possible to predict the existence of a weakly interacting fermion of rest energy $584.725 \mathrm{GeV}$, called Higg's fermion. Cosmological 'dark matter' research and observations can be carried out in this direction also.

\section{Three Assumptions}

With reference to our recent publications [7-12], we propose the following three assumptions:

(1) There exist four different gravitational constants associated with gravitational, weak, electromagnetic and strong interactions.

Let, Newtonian gravitational constant $=G_{N}$

Electromagnetic gravitational constant $={ }_{e}$

Nuclear gravitational constant $=G_{s}$

Weak gravitational constant $=G_{w}$ 


\section{A short communication}

$G_{e} \cong 2.374335 \times 10^{37} \mathrm{~m}^{3} \mathrm{~kg}^{-1} \mathrm{sec}^{-2}$
$G_{s} \cong 3.329561 \times 10^{28} \mathrm{~m}^{3} \mathrm{~kg}^{-1} \mathrm{sec}^{-2}$
$G_{w} \cong 2.909745 \times 10^{22} \mathrm{~m}^{3} \mathrm{~kg}^{-1} \mathrm{sec}^{-2}$
$G_{N} \cong 6.679855 \times 10^{-11} \mathrm{~m}^{3} \mathrm{~kg}^{-1} \mathrm{sec}^{-2}$
(See point-7 of 'Discussion'
for the supporting relations).

(2) Characteristic nuclear radius can be expressed with, $R_{0} \cong \frac{2 G_{s} m_{p}}{c^{2}} \cong 1.24 \mathrm{fm}$

(3) There exists a strong elementary charge $\left(e_{s}\right)$ in such a way that,

$$
\begin{gathered}
\frac{m_{p}}{m_{e}} \cong\left(\frac{e_{s}^{2}}{4 \pi \varepsilon_{0} G_{s} m_{p}^{2}}\right) /\left(\frac{e^{2}}{4 \pi \varepsilon_{0} G_{e} m_{e}^{2}}\right) \cong\left(\frac{G_{s} m_{p}^{2}}{\hbar c}\right)\left(\frac{G_{e} m_{e}^{2}}{\hbar c}\right) \\
\rightarrow\left\{\begin{array}{l}
\frac{e_{s}^{2}}{e^{2}} \cong\left(\frac{G_{s} m_{p}^{3}}{G_{e} m_{e}^{3}}\right) \cong\left(\frac{G_{s} m_{p}^{2}}{\hbar c}\right)^{2} \cong \frac{1}{\alpha_{s}} \\
\frac{e_{s}}{e} \cong \sqrt{\frac{G_{s} m_{p}^{3}}{G_{e} m_{e}^{3}} \cong\left(\frac{G_{s} m_{p}^{2}}{\hbar c}\right) \cong \sqrt{\frac{1}{\alpha_{s}}}}
\end{array}\right.
\end{gathered}
$$

where, $\alpha_{s} \cong$ Strong coupling constant

Based on these assumptions,

$$
\begin{aligned}
& e_{s} \cong 2.9463591 e \\
& \alpha_{s} \cong 0.1151937 \\
& \frac{1}{\alpha_{s}} \cong 8.681032 \\
& R_{0} \cong 1.23929 \times 10^{-15} \mathrm{~m}
\end{aligned}
$$

4. Understanding proton-neutron stability with three atomic gravitational constants

$$
\begin{aligned}
& s \cong\left\{\left(\frac{e_{s}}{m_{p}}\right) \div\left(\frac{e}{m_{e}}\right)\right\} \cong 0.001605 \\
& \left.\cong \sqrt{\frac{G_{s} m_{p}}{G_{e} m_{e}}} \cong \frac{G_{s} m_{p} m_{e}}{\hbar c} \cong \frac{\hbar c}{G_{e} m_{e}^{2}} \cong \frac{G_{s}^{2}}{G_{e} G_{w}} \cong \frac{m_{p}}{M_{w}}\right\} \\
& \text { where, } M_{w} \cong \sqrt{\hbar c / G_{w}} \cong 584.725 \mathrm{GeV} / c^{2}
\end{aligned}
$$

Nuclear beta stability line can be addressed with a relation of the form,

$$
\begin{aligned}
& A_{s} \cong Z+N_{s} \\
& \cong 2 Z+s(2 Z)^{2} \cong 2 Z+(4 s) Z^{2} \\
& \cong 2 Z+k Z^{2} \cong Z(2+k Z)
\end{aligned}
$$

where $k \cong 4 s \cong 0.0064185$

By considering a factor like $[2 \pm \sqrt{k}]$, likely possible range of $A_{s}$ can be addressed with,

$$
\left\{\begin{array}{l}
\left(A_{s}\right)_{\text {lower }} \cong Z(1.92+k Z) \\
\left(A_{s}\right)_{\text {mean }} \cong Z(2.0+k Z) \\
\left(A_{s}\right)_{\text {upper }} \cong Z(2.08+k Z)
\end{array}\right\}
$$

\section{Understanding nuclear binding energy}

(1) $B_{0} \cong \frac{e_{s}^{2}}{4 \pi \varepsilon_{0} R_{0}} \cong \frac{e_{s}^{2}}{8 \pi \varepsilon_{0}\left(G_{s} m_{p} / c^{2}\right)} \cong 10.09 \mathrm{MeV}$ can be considered as the unique binding energy coefficient.

(2) With reference to $a_{c}=0.71 \mathrm{MeV}$ and $a_{a}=23.21 \mathrm{MeV}$, close to stable mass numbers, binding energy seems to be proportional to $\left(1-2\left(a_{c} / a_{a}\right)^{2} \sqrt{Z N}\right) A \cong(1-0.00189 \sqrt{Z N}) A$

where $2\left(a_{c} / a_{a}\right)^{2} \cong 0.0018753 \cong 0.00189$. The ad-hoc coefficient 0.00189 somehow, seems to lie in between $\{s \cong 0.0016$ and $k \cong 0.0064\}$. With reference to electromagnetic interaction, we consider, $[k / \ln (30)] \cong 0.00189$ where 30 is a characteristic representation of atomic number below which strength of nuclear binding energy $[7,10,11,12]$ seems to decrease by $[Z / 30]^{\sqrt{k}}\left(1 / \alpha_{s}\right) \cong[Z / 30]^{0.08} \times 8.68$. From $Z=30$ onwards, strength of nuclear binding energy remains same at $\left(1 / \alpha_{s}\right) \cong 8.68$. See point 4 of 'Discussion'. It needs further study.

(3) Binding energy can be assumed to decrease with increasing radius.

(4) Decreasing proton-neutron ratio seems to play an interesting role in increasing binding energy.

(5) Considering isotopes, stable mass number plays an interesting role in estimating the binding energy of $A$ in the form of $\left(\left(A_{s}-A\right)^{2} / A_{s}\right)$.

Based on these points and considering light, medium and heavy atomic nuclides, for $(\mathrm{Z} \approx 3$ to 118$)$, close to the stable mass number, 


\section{A short communication}

$$
B_{A_{s}} \cong\left\{\left(1-0.00189 \sqrt{Z N_{s}}\right) A_{s}-A_{s}^{1 / 3}-\left(\frac{Z}{N_{s}}\right)\right\} B_{0}
$$

See Figure 1. Dashed red curve plotted with relations (2) and (4) can be compared with the green curve plotted with the standard SEMF. For light, medium and heavy atomic nuclides, fit is reasonable.

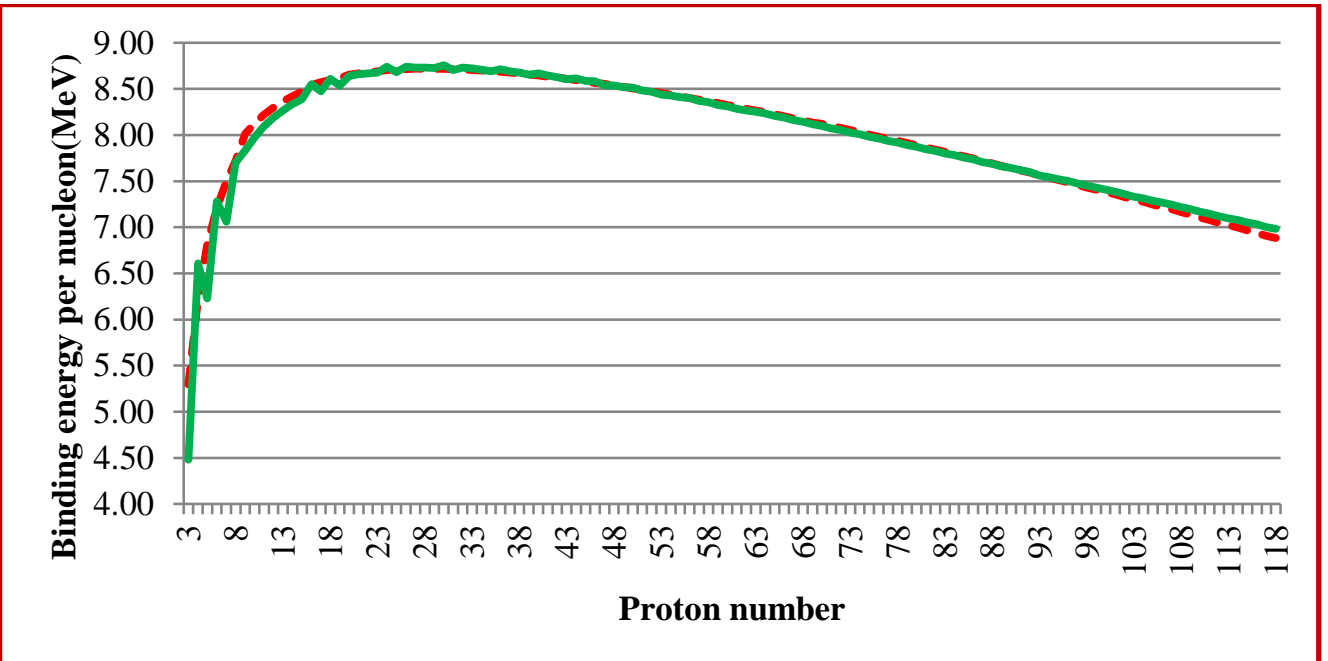

Figure 1: Binding energy per nucleon close to stable mass numbers of $Z=3$ to 118

Above and below the stable mass numbers, binding energy can be approximated with,

$$
B_{A} \cong\left\{\begin{array}{r}
(1-0.00189 \sqrt{Z N}) A-A^{1 / 3} \\
-\left(\frac{Z}{N}\right)-\frac{\left(A_{s}-A\right)^{2}}{A_{s}}
\end{array}\right\} B_{0}
$$

See Figure 2 for the estimated isotopic binding energy of $\mathrm{Z}=50$. Dotted blue curve plotted with relations (2) and (5) can be compared with the green curve plotted with SEMF. Based on Figures 1 and 2, it is possible to say that,
1) Relations (2) and (5) can also be given some priority in understanding nuclear binding energy scheme.

2) Estimated binding energy can also be compared with spherical relativistic continuum HartreeBogoliubov (RCHB) theory data [2] and ThomasFermi model (Table of nuclear masses, nsdssd.lbl.gov, 1994).

3) For $(N<Z)$ and $(N \approx Z)$ estimated binding energy seems to be increasing compared to SEMF estimation.

4) For $\left(A>>A_{s}\right)$, estimated binding energy seems to be decreasing compared to SEMF estimation.

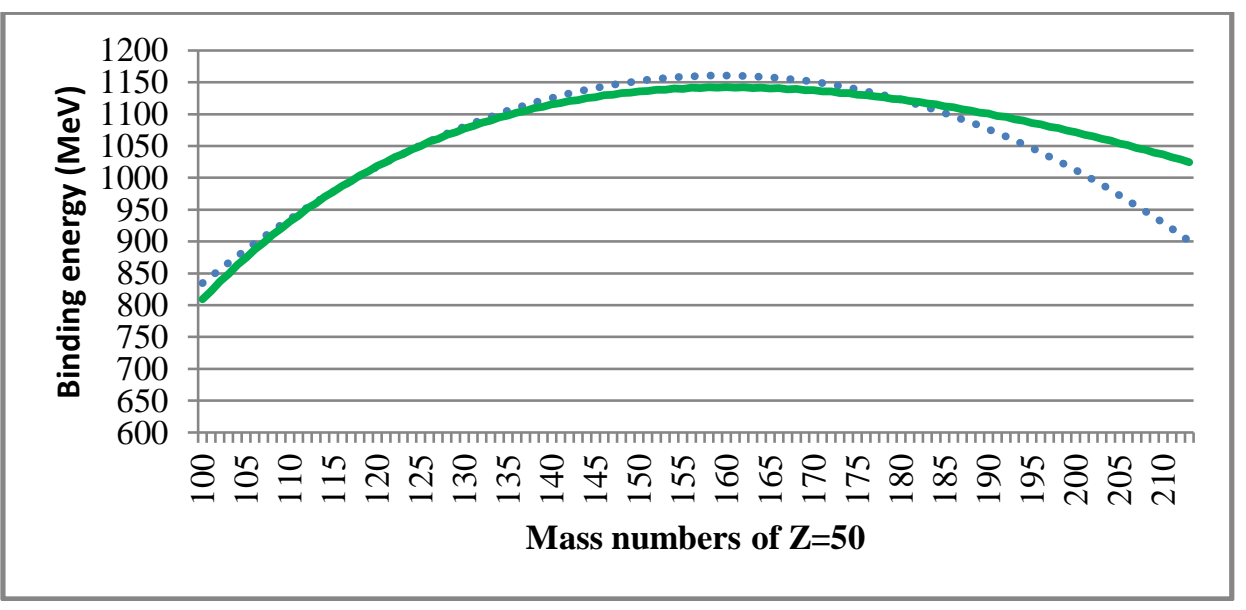




\section{A short communication}

Figure 2: Isotopic binding energy of $Z=50$

\section{Discussion}

(1) Nuclear binding energy can be understood with a single and unified energy coefficient.

(2) The new numbers $(s, k)$ seem to play an interesting role in understanding nuclear stability and binding energy.

(3) Considering a term of the form $\left(1-0.00189 \sqrt{Z \sqrt{N N_{s}}}\right)$ or by modifying the terms, $\quad(Z / N)$ and $\left(\left(A_{s}-A\right)^{2} / A_{s}\right)$ binding energy for $\left(A<<A_{s}\right)$ and $\left(A \gg>A_{s}\right)$ can be understood.

(4) $\mathrm{Z}=(2$ to 118$)$, close to stable mass numbers, binding energy [10] can also be approximated with,

For $Z<30$ and $A_{s} \cong Z(2+k Z)$,

$\left(B_{A_{s}}\right) \cong\left(\frac{Z}{30}\right)^{0.08}\left\{A_{s}-\left[\left(0.00189 N_{s}^{2}\right)+\frac{1}{2}\right]\right\} 9.16 \mathrm{MeV}$

For $Z \geq 30$ and $A_{s} \cong Z(2+k Z)$,

$\left(B_{A_{s}}\right) \cong\left\{A_{s}-\left[\left(0.00189 N_{s}^{2}\right)+\frac{1}{2}\right]\right\} 9.16 \mathrm{MeV}$

where, $\left\{\begin{array}{l}\frac{e_{s}^{2}}{8 \pi \varepsilon_{0}\left(G_{s} m_{p} / c^{2}\right)}-\left(\frac{e^{2}}{4 \pi \varepsilon_{0} R_{0}}\right) \cong 8.928 \mathrm{MeV} \\ \frac{e_{s}^{2}}{8 \pi \varepsilon_{0}\left(G_{s} m_{p} / c^{2}\right)}-\frac{3}{5}\left(\frac{e^{2}}{4 \pi \varepsilon_{0} R_{0}}\right) \cong 9.395 \mathrm{MeV} \\ \text { and } \frac{8.928+9.395}{2} \cong 9.16 \mathrm{MeV}\end{array}\right\}$

(5) In case of Deuteron, there exists no strong interaction in between proton and neutron $[7,11]$.

(6) Nuclear charge radii [2,5] can be addressed with,

$$
R_{(Z, A)} \cong\left\{Z^{1 / 3}+(\sqrt{Z(A-Z)})^{1 / 3}\right\}\left(\frac{G_{s} m_{p}}{c^{2}}\right)
$$

(7) The following set of four semi empirical relations can be considered as REFERENCE relations [912]. They need further investigation.

$$
\left.\begin{array}{rl}
\text { A) } \frac{m_{p}}{m_{e}} \cong 2 \pi \sqrt{\frac{4 \pi \varepsilon_{0} G_{e} m_{e}^{2}}{e^{2}}} \\
\text { B) } \hbar c \cong\left(\frac{m_{p}}{m_{e}}\right)^{2}\left(G_{e}^{2} G_{N}\right)^{1 / 3} m_{p}^{2} \\
\text { C) } \frac{G_{w}}{G_{N}} \cong\left(\frac{m_{p}}{m_{e}}\right)^{10} \\
\text { D) } G_{F} \cong \text { Fermi'sWeak coupling constant } \\
\cong\left[\left(G_{e} m_{p}^{2}\right)^{2}\left(G_{N} m_{p}^{2}\right)\right]^{\frac{1}{3}}\left(\frac{2 G_{s} m_{p}}{c^{2}}\right)^{2} \\
\cong \frac{4 G_{w} \hbar^{2}}{c^{2}} \cong 1.44021 \times 10^{-62} \mathrm{~J} \cdot \mathrm{m}^{3}
\end{array}\right\}
$$

\section{Conclusion}

Understanding nuclear binding energy with single energy coefficient in terms of fundamental interactions is a very challenging task. In this context, we tried our level best in presenting a very simple and effective semi empirical formula with one unique energy coefficient. It needs further investigation.

Role played by the four gravitational constants seems to be fairly natural. By implementing four such gravitational constants in String theory models, it may be possible to explore the hidden unified physics. Proceeding further, theoretical value of $G_{N}$ can be defined as a standard reference for future nuclear, atomic and gravitational experiments.

\section{References}

[1] Cht. Mavrodiev S, Deliyergiyev MA. Modification of the nuclear landscape in the inverse problem framework using the generalized Bethe-Weizsäcker mass formula. Int. J. Mod. Phys. E27: 1850015 (2018)

[2] Xiaa XW, et al. The limits of the nuclear landscape explored by the relativistic continuum Hatree-Bogoliubov theory. Atomic Data and Nuclear Data Tables. 121-122:1-215. (2018)

[3] Ghahramany $\mathrm{N}$ et al. New approach to nuclear binding energy in integrated nuclear model. Journal of Theoretical and Applied Physics. 6:3 (2012)

[4] Seshavatharam UVS et al. Understanding the constructional features of materialistic atoms in the light of strong nuclear gravitational coupling. Materials Today: $3 / 10 \mathrm{~PB}$, Proceedings 3, 3976-3981 (2016)

[5] Seshavatharam UVS and Lakshminarayana S. Towards a workable model of final unification. International Journal of Mathematics and Physics 7(1), 117-130 (2016)

[6] Seshavatharam UVS and Lakshminarayana S. Understanding the basics of final unification with three gravitational constants associated with nuclear, electromagnetic and gravitational interactions. Journal of Nuclear Physics, Material Sciences, Radiation and Applications 4(1),1-19 (2017)

[7] Seshavatharam UVS and Lakshminarayana S. On the role of 'reciprocal' of the strong coupling constant in nuclear structure. Journal of Nuclear Sciences, 4( 2), 31-44 (2017).

[8] Seshavatharam UVS and Lakshminarayana S. Fermi scale 


\section{A short communication}

applications of strong (nuclear) gravity-1. Proceedings of the DAE Symp. on Nucl. Phys. 63, 72-73. (2018)

[9] Seshavatharam UVS and Lakshminarayana S. A virtual model of microscopic quantum gravity, Prespacetime Journal, 9(1), 58-82 (2018).

[10] Seshavatharam UVS and Lakshminarayana S. On the Role of Squared Neutron Number in Reducing Nuclear Binding Energy in the Light of Electromagnetic, Weak and Nuclear Gravitational Constants - A Review. Asian Journal of Research and Reviews in Physics, 2(3): 1-22, (2019)

[11] Seshavatharam UVS and Lakshminarayana S. On the role of four gravitational constants in nuclear structure. Mapana Journal of Sciences, 18(1), 21-45 (2019)

[12] Seshavatharam UVS and Lakshminarayana S. On the Role of Large Nuclear Gravity in Understanding Strong Coupling Constant, Nuclear Stability Range, Binding Energy of Isotopes and Magic proton numbers - A Critical Review. J. Nucl. Phys. Mat. Sci. Rad. A. 6(2), 142-160 (2019) 